\title{
Characteristics of New Opioid Use Among Medicare Beneficiaries: Identifying High-Risk Patterns
}

Sudha R. Raman, PhD; Christopher Bush, MPH; Ruchir N. Karmali, PhD; Lawrence H. Greenblatt, MD; Andrew W. Roberts, PharmD, PhD; and Asheley C. Skinner, PhD

\begin{abstract}
BACKGROUND: Opioid prescription patterns, including long-term use, multiple prescribers, and high opioid doses, increase the risk for adverse outcomes; however, previous research in older adult populations has primarily described opioid dose patterns using average daily dose measures or using very high thresholds (i.e., > 100 morphine milligram equivalents [MME] per day).

OBJECTIVE: To describe prescription patterns by peak dose among older adults who have newly initiated opioid use in 2014 and describe long-term opioid use and the use of multiple pharmacies and prescribers among those with peak opioid doses over 50 and over 90 MME per day.

METHODS: This was a retrospective cohort study of Medicare Part D prescription claims data ( $5 \%$ sample) for beneficiaries aged 65 years and older who were prescribed $\geq 1$ opioid prescription in 2014 and did not have an opioid prescription in the preceding 180 days. Within a 1-year period of follow-up, we used prescription claims to characterize individuals' opioid exposure, measuring long-term opioid use ( $\geq 90$ days of continuous opioid supply), unique opioid prescribers, and unique opioid-dispensing pharmacies. Peak MME was defined as the maximum daily MME received across all overlapping opioid prescriptions in the observation period.
\end{abstract}

RESULTS: 144,127 beneficiaries without an opioid prescription in the previous 6 months filled $\geq 1$ opioid prescription in 2014. During the 1-year follow-up period, $6.5 \%$ of beneficiaries transitioned to long-term opioid use; $39.5 \%$ received opioid prescriptions from $>1$ prescriber; $18.1 \%$ filled opioid prescriptions from $>1$ pharmacy; and $21.8 \%$ had a peak MME of 50-89. Among the $28.1 \%$ of beneficiaries exposed to a peak MME $\geq 50,8.6 \%$ developed long-term opioid use; $7.0 \%$ had 3 or more opioid dispensing pharmacies; and $28.0 \%$ had 3 or more opioid prescribers. Among the $6.2 \%$ of beneficiaries exposed to a peak MME $\geq 90,18.5 \%$ developed long-term opioid use; $13.0 \%$ had 3 or more opioid dispensing pharmacies; and $39.6 \%$ had 3 or more opioid prescribers.

CONCLUSIONS: High doses of opioids were prescribed for about one quarter (28\%) of Medicare beneficiaries with new opioid use in 2014. Having multiple opioid prescribers or multiple opioid dispensing pharmacies was common, especially among those prescribed higher doses. These prescription patterns can be particularly helpful to identify older adults with increased opioid-related risk.

J Manag Care Spec Pharm. 2019;25(9):966-72

Copyright $\odot 2019$, Academy of Managed Care Pharmacy. All rights reserved.

\section{What is already known about this subject}

Certain opioid prescription patterns, such as type of opioid, multiple prescribers, and multiple pharmacies, are associated with an increased risk for adverse opioid-related outcomes.

Opioid dose patterns among older adults have been described using average daily dose measures or very high thresholds.

\section{What this study adds}

Over one quarter of Medicare beneficiaries aged 65 years and older with new opioid use received peak morphine milligram equivalents (MME) doses of opioids more than $50 \mathrm{MME}$ (the lowest guideline threshold from the Centers for Disease Control and Prevention)

Among Medicare beneficiaries who have been prescribed higher doses (defined as either $>50 \mathrm{MME}$ or $>90 \mathrm{MME}$ ), having multiple opioid prescribers or multiple opioid dispensing pharmacies was common.

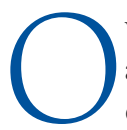
ver half of adults aged 65 years and older experience a painful condition; as such, the use of prescription opioids is common among older adults. ${ }^{1}$ From 1999 to 2010, the proportion of outpatient clinic visits resulting in an opioid prescription increased from $4 \%$ to $9 \% .^{2}$ In 2017, despite stable or declining prescription rates, 1 in 5 older adults in the United States had an opioid prescription. ${ }^{3}$ Opioid-related mortality in this group continues to rise each year, as do nonfatal adverse outcomes such as opioid-related emergency room visits, hospital admissions, and opioid misuse. . $^{4-7}$

Certain opioid prescription patterns are associated with opioid-related harms in older adult populations, including type of opioid, number of days of opioid use, and multiple opioid prescribers. ${ }^{8-10}$ In 2012, 3\% of Medicare beneficiaries had very high doses ( $\geq 120$ morphine milligram equivalents [MME]); about $7 \%$ of older adult Medicare beneficiaries had long-term opioid use ( $\geq 90$ days supply); and intensive use of opioids (defined as $\geq 6$ fills or use for $\geq 90$ consecutive days) occurred in about $10 \%-13 \%$ of Medicare beneficiaries. ${ }^{10-12}$ Two thirds of Medicare beneficiaries who had been prescribed opioid medication had multiple opioid prescribers, which may, in some cases, reflect "doctor shopping" or a fragmented care system. ${ }^{9}$ 
Given the inherent clinical risk that opioids pose in this population, and the frequency of prescription opioid use among older adults, dose may be an important modifiable risk factor. Prescribed daily opioid quantity has a dose-response relationship with opioid-related death and nonfatal overdose. ${ }^{13}$ Previous estimates of average MME across a time period may also mask short periods of high-risk and high-intensity doses. Studies of older adults have not examined dose in the context of other opioid prescribing patterns, such as long-term use or multiple prescribers, or have used very high MME thresholds (e.g., > 120 MME per day)..$^{9-12}$

Our objective was to describe the prevalence of high-risk opioid use patterns, including peak opioid doses exceeding the guideline threshold of $50 \mathrm{MME}$ per day from the Centers for Disease Control and Prevention (CDC), long-term use, and the use of multiple pharmacies or prescribers among older adult Medicare beneficiaries newly initiating opioid treatment.

\section{Methods}

Medicare provides health insurance coverage for nearly all of the U.S. population aged 65 years and older, and more than $70 \%$ of Medicare beneficiaries receive prescription drug coverage from a Medicare Part D prescription drug plan. ${ }^{14}$ Our cohort was selected from a 5\% national sample of beneficiaries in the fee-for-service (FFS) Medicare program (not Medicare Advantage program) and used data from the Medicare Master Beneficiary Summary File and Part D prescription claims data from 2013 to 2015.

The study population included Medicare beneficiaries aged 65 years and older who qualified for Medicare based on age, were enrolled in Medicare Part D, and had a record of $\geq 1$ opioid prescription in 2014. We identified individuals newly initiating prescription opioid treatment in order to include those who were relatively opioid naive and to exclude prevalent users, who may have been a group with heterogeneous medication use patterns. Study follow-up started at the index date, defined as the date of the first opioid prescription in 2014 following $\geq 180$ days without a claim for an opioid prescription; the 180 day period was defined similar to previous research. ${ }^{15}$ We required individuals to have 1 year of follow-up from the index date and excluded individuals if they had evidence of hospice service use, did not have continuous FFS or Part D coverage, or died during the 1-year follow-up period. The exclusion of individuals with active or historical cancer from opioid research is a growing concern,${ }^{16}$ since this group has numerous risk factors for opioid misuse, opioid use disorders, and overdose. ${ }^{17-19}$ Therefore, we did not exclude individuals with cancer diagnoses so that we could obtain a 1-year estimate of opioid use in this population for any indication. Opioid exposure was defined as a prescription claim for an oral or transdermal opioid analgesic with an indication to treat pain that was approved by the U.S. Food and Drug Administration.
Demographic characteristics were derived from the Medicare Master Beneficiary Summary File and included age, sex, race, and Medicaid dual eligibility (an indication of low-income subsidy and a proxy variable for income). ${ }^{20}$

\section{Opioid Use Measures}

Measures for opioid use patterns over 1 year were constructed from Medicare Part D prescription claims records. MME was calculated for each prescription based on National Drug Code numbers and MME conversions factors provided by the CDC. ${ }^{21}$ Daily MME exposure was assessed for each beneficiary by summing MME from all overlapping opioid prescriptions on a given day. Peak MME was defined as the maximum calculated daily MME received during the observation period and represented the day with the highest "as prescribed" dose. Average daily MME was defined as the patient's total MME from all prescriptions received during follow-up divided by the total available days supply from all prescriptions. If a beneficiary's prescription was filled before the end of the previous prescription, the overlapping days were only counted once in the denominator.

For each included beneficiary, we measured the number of unique opioid prescribers and unique opioid-dispensing pharmacies and identified the type of opioid products received, including long-acting and short-acting Schedule II and III products. Long-term opioid use was defined as having $\geq 90$ days of continuous opioid supply, with gaps of no more than 7 days between consecutive prescriptions.

\section{Statistical Analysis}

Descriptive statistics are presented for the study cohort by peak MME, which was categorized according to CDC guidelines for opioid exposure (<20 MME, 20-49 MME, 50-89 MME, and $\geq 90 \mathrm{MME}$, as well as $>50 \mathrm{MME})^{22}$ Frequencies with proportions are used to describe categorical opioid-prescribing patterns and demographic characteristics, while means with standard deviations are provided for continuous measures. Given the size of the cohort, even very small differences were statistically significant, so we do not present $P$ values; instead, we encourage readers to consider the clinical and public health significance of reported values.

Analyses were conducted with SAS version 9.4 (SAS Institute, Cary, NC). The Duke University School of Medicine Institutional Review Board approved this study.

\section{Results}

We identified 328,688 older adult Medicare beneficiaries who filled $\geq 1$ prescription for an opioid in 2014. After excluding 160,213 beneficiaries who received an opioid prescription during the 6 months preceding the 2014 index date, and 24,348 beneficiaries without 1 year of follow-up (including 19,015 who 


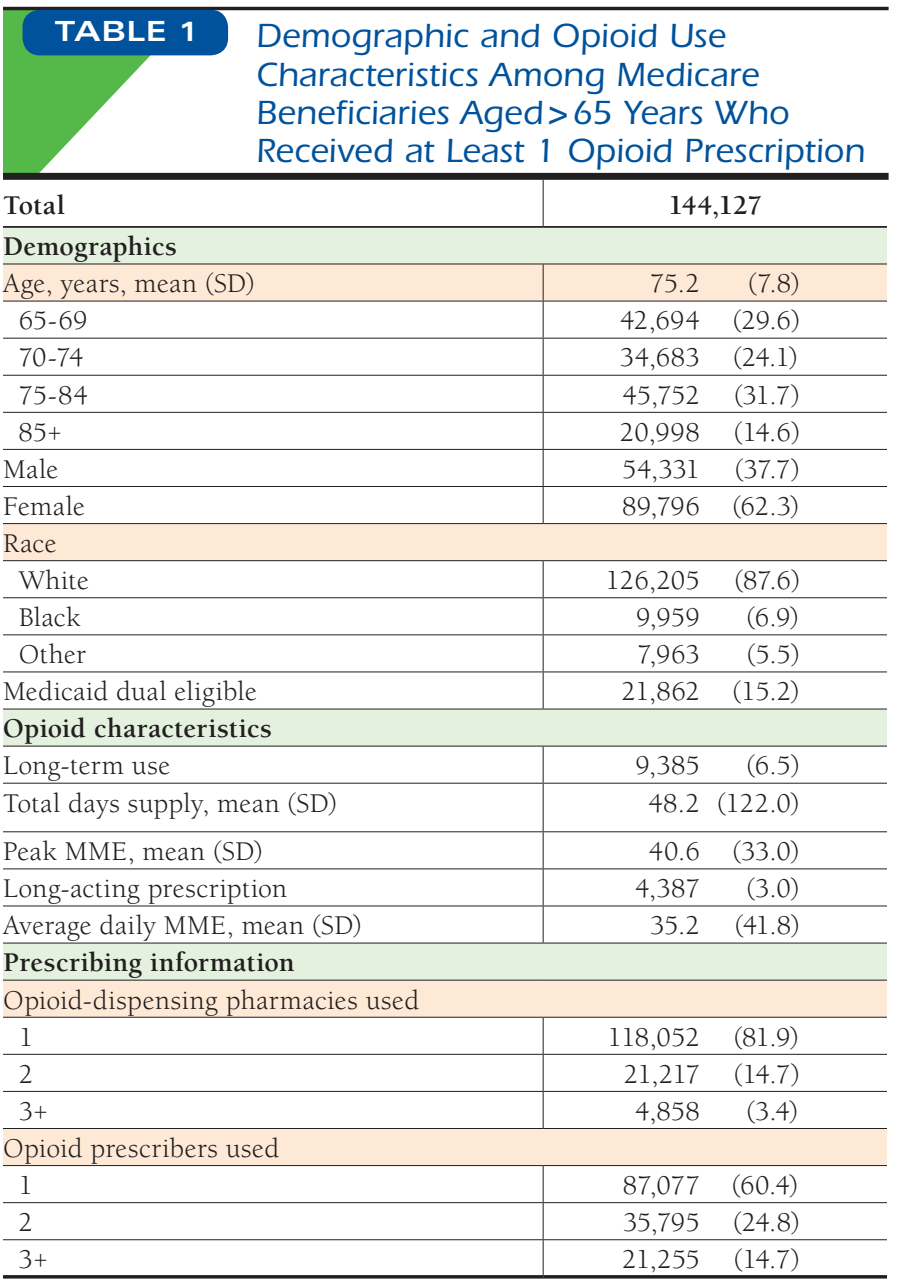

Note: Number (\%) unless otherwise indicated.

$M M E=$ milligram morphine equivalents; $S D=$ standard deviation

died), the final study cohort included 144,127 beneficiaries with new prescription opioid use in 2014.

Of the study cohort, the average age was 75 years; $62.3 \%$ were female; and $87.6 \%$ were white. Medicaid dual eligibility was present in $15.2 \%$ of beneficiaries. In the year following their index opioid prescriptions, $6.5 \%$ of beneficiaries transitioned to long-term opioid use; $18.1 \%$ filled opioid prescriptions from more than 1 pharmacy, and $39.6 \%$ received opioid prescriptions from more than 1 prescriber. The mean daily MME average was 35.2, and the mean peak MME per day was 40.6 (Table 1).

Of these older adult Medicare beneficiaries with new opioid use, most (56.2\%) had a peak MME of 20-49 per day during the year of follow-up; $21.8 \%$ had a peak MME of 50-89 per day; and $6.2 \%$ had a peak MME $>90$ per day. Compared with other peak MME categories, those in the highest peak MME categories were more likely to be younger; $42.9 \%$ of those with $\geq 90$ MME per day (37.4\% of those with $50+$ MME per day) were aged 65-69 years, compared with $22.0 \%$ in the $<20$ MME per day category. They were also more likely to be male and white and less likely to be eligible for Medicaid coverage (Table 2).

Individuals with peak $\mathrm{MME} \geq 50$ per day had a greater proportion of long-term use (8.6\%) compared with individuals in lower peak MME categories (5\%-8.1\%). Of the $28 \%$ of beneficiaries who were classified into the peak $M M E \geq 50$ per day group, $7.8 \%$ had $\geq 1$ long-acting prescription; $7 \%$ had 3 or more opioid-dispensing pharmacies; and $28 \%$ had 3 or more opioid prescribers. Similarly, individuals with peak MME $\geq 90$ per day had a greater proportion of long-term use (18.5\%) compared with individuals in lower peak MME categories (5\%-8\%). Of the $6.2 \%$ of beneficiaries who were classified into the peak $\mathrm{MME} \geq 90$ per day group, $19.3 \%$ had $\geq 1$ long-acting prescription; $13.0 \%$ had $3+$ opioid-dispensing pharmacies, and $39.6 \%$ had $3+$ opioid prescribers; each of these was much less common among those with lower peak MME.

\section{Discussion}

The results of this study provide 3 novel insights about prescription opioid use patterns among older adult Medicare beneficiaries. First, over 1 in 4 beneficiaries were exposed to a peak $\mathrm{MME} \geq 50$ in the year following initiation of new opioid therapy; $50 \mathrm{MME}$ was recognized as a commonly used threshold for increased risk for opioid harm. ${ }^{22}$ Second, we observed that 1 of 15 beneficiaries with new opioid use with a prescription of any length transitioned to long-term opioid use, while nearly 1 in 5 who received a peak MME $\geq 90$ also had long-term use. Third, although the majority of beneficiaries used a single opioid-dispensing pharmacy and a single opioid-prescribing provider, having multiple opioid prescribers or multiple opioiddispensing pharmacies was common among those receiving higher doses ( $\geq 50$ or 90 MME per day).

A particularly worrisome finding is that in the year following their index prescription dates, more than one quarter of beneficiaries experienced opioid exposure with a peak MME that would be considered high risk according to CDC guidelines. Opioid-prescribing guidelines in effect during the study period focused on higher dose thresholds (over $100 \mathrm{MME}$ ). ${ }^{23}$ The CDC's opioid prescription guidelines, which were published in 2016 (after the study period), recommend initiating opioid treatment with the lowest effective dose, careful assessment of the benefits and risks when increasing dosage to $\geq 50$ MME, and avoiding or carefully justifying dosages of $\geq 90$ MME. ${ }^{22}$ Opioid prescription guidelines for older adults from the American Geriatrics Society make similar recommendations without specific dose thresholds. ${ }^{24}$ 


\section{TABLE 2 Demographic and Opioid Use Characteristics by Categories of Peak MME}

\begin{tabular}{|c|c|c|c|c|c|c|c|c|c|c|}
\hline \multirow[b]{2}{*}{ Number } & \multicolumn{2}{|c|}{$<20 \mathrm{MME}$} & \multicolumn{2}{|c|}{ 20-49 MME } & \multicolumn{2}{|c|}{ 50-89 MME } & \multicolumn{2}{|c|}{$\geq 90 \mathrm{MME}$} & \multicolumn{2}{|c|}{$>50 \mathrm{MME}$} \\
\hline & 22,691 & $(15.7)$ & 80,947 & $(56.2)$ & 31,473 & $(21.8)$ & 9,016 & $(6.2)$ & 40,489 & $(28.1)$ \\
\hline \multicolumn{11}{|l|}{ Demographics } \\
\hline Age, years, mean (SD) & 77.4 & $(8.4)$ & 75.6 & $(7.8)$ & 73.4 & $(6.8)$ & 72.2 & $(6.4)$ & 73.2 & $(6.8)$ \\
\hline $65-69$ & 4,989 & $(22.0)$ & 22,543 & $(27.8)$ & 11,295 & $(35.9)$ & 3,867 & $(42.9)$ & 15,162 & $(37.4)$ \\
\hline $70-74$ & 4,656 & $(20.5)$ & 19,048 & $(23.5)$ & 8,527 & $(27.1)$ & 2,452 & $(27.2)$ & 10,979 & $(27.1)$ \\
\hline $75-84$ & 7,772 & $(34.3)$ & 26,766 & $(33.1)$ & 9,028 & $(28.7)$ & 2,186 & $(24.2)$ & 11,214 & $(27.7)$ \\
\hline $85+$ & 5,274 & (23.2) & 12,590 & (15.6) & 2,623 & (8.3) & 511 & $(5.7)$ & 3,134 & $(7.7)$ \\
\hline Male & 6,835 & $(30.1)$ & 29,831 & (36.9) & 13,627 & (43.3) & 4,038 & (44.8) & 17,665 & (43.6) \\
\hline Female & 15,856 & $(69.9)$ & 51,116 & $(63.1)$ & 17,846 & $(56.7)$ & 4,978 & $(55.2)$ & 22,824 & $(56.4)$ \\
\hline \multicolumn{11}{|l|}{ Race } \\
\hline White & 18,577 & (81.9) & 70,856 & $(87.5)$ & 28,538 & (90.7) & 8,234 & (91.3) & 36,772 & $(90.8)$ \\
\hline Black & 2,194 & $(9.7)$ & 5,806 & $(7.2)$ & 1,534 & (4.9) & 425 & $(4.7)$ & 1,959 & $(4.8)$ \\
\hline Other & 1,920 & $(8.5)$ & 4,285 & $(5.3)$ & 1,401 & $(4.5)$ & 357 & $(4.0)$ & 1,758 & $(4.3)$ \\
\hline Medicaid dual eligible & 5,362 & $(23.6)$ & 12,285 & $(15.2)$ & 3,239 & $(10.3)$ & 976 & $(10.8)$ & 4,215 & $(10.4)$ \\
\hline \multicolumn{11}{|l|}{ Opioid characteristics } \\
\hline Long-term use & 1,837 & $(8.1)$ & 4,051 & $(5.0)$ & 1,825 & $(5.8)$ & 1,672 & $(18.5)$ & 3,497 & $(8.6)$ \\
\hline Total days supply, mean (SD) & 56.4 & $(119.7)$ & 39.5 & $(100.4)$ & 44.3 & $(121.3)$ & 119.2 & $(232.0)$ & 60.9 & $(156.2)$ \\
\hline Peak MME, mean (SD) & 13.5 & $(4.2)$ & 31.3 & $(8.2)$ & 61.0 & $(10.6)$ & 120.7 & $(76.6)$ & 74.3 & $(44.8)$ \\
\hline Long-acting prescription & 130 & $(0.6)$ & 1,087 & $(1.3)$ & 1,429 & $(4.5)$ & 1,741 & (19.3) & 3,170 & $(7.8)$ \\
\hline Average daily MME, mean (SD) & 13.9 & $(8.9)$ & 29.1 & $(16.5)$ & 49.3 & $(27.5)$ & 94.1 & $(130.5)$ & 59.3 & $(68.7)$ \\
\hline \multicolumn{11}{|c|}{ Prescribing information } \\
\hline \multicolumn{11}{|c|}{ Opioid-dispensing pharmacies used } \\
\hline 1 & 20,996 & $(92.5)$ & 68,408 & $(84.5)$ & 23,254 & $(73.9)$ & 5,394 & (59.8) & 28,648 & (70.8) \\
\hline 2 & 1,511 & $(6.7)$ & 10,717 & $(13.2)$ & 6,535 & $(20.8)$ & 2,454 & $(27.2)$ & 8,989 & $(22.2)$ \\
\hline 3 & 184 & $(0.8)$ & 1,822 & $(2.3)$ & 1,684 & $(5.4)$ & 1,168 & $(13.0)$ & 2,852 & $(7.0)$ \\
\hline \multicolumn{11}{|l|}{ Opioid prescribers used } \\
\hline 1 & 19,264 & $(84.9)$ & 50,993 & $(63.0)$ & 14,023 & $(44.6)$ & 2,797 & $(31.0)$ & 16,820 & $(41.5)$ \\
\hline 2 & 2,818 & $(12.4)$ & 20,625 & $(25.5)$ & 9,702 & $(30.8)$ & 2,650 & $(29.4)$ & 12,352 & $(30.5)$ \\
\hline 3 & 609 & $(2.7)$ & 9,329 & (11.5) & 7,748 & (24.6) & 3,569 & (39.6) & 11,317 & $(28.0)$ \\
\hline
\end{tabular}

Note: Number (\%) unless otherwise indicated.

$M M E=$ milligram morphine equivalents; $S D=$ standard deviation

Considering that the assessment and treatment of pain among older adults may be complicated by comorbidities, other medications, and uncertainty about how to implement opioid-prescribing guidelines in a heterogenous population, our results add to the literature by examining the prevalence of this lowest dose threshold at which risk may increase among a population of opioid-naive Medicare beneficiaries. ${ }^{24}$

Our findings are of greater concern in light of how we defined peak MME. This measure was designed to understand opioid exposure, considering all concurrent opioid prescriptions regardless of their source or indication. Prescription-level average MME measures may mask MME peaks that place individuals at greater risk of overdose or other negative outcomes. Although more work is needed to compare how peak MME and average MME predict opioid-related harms, the concept of peak MME may be particularly important for those with multiple prescribers, where an individual prescriber follows guidelines for a patient, but the total from all prescribers places the patient at greater risk. The risk that is associated with these high doses may be preventable, in particular, if our findings about multiple providers are due to uncoordinated or fragmented care.

The frequency of multiple opioid prescribers and long-term use, which are risk factors for opioid-related adverse outcomes, were similar to previous research on this population. ${ }^{10}$ Of note, these risk factors for opioid-related adverse outcomes were more common among those with high dosages (> $50 \mathrm{MME}$ and $>90 \mathrm{MME}$ ). The appropriateness of long-term opioid use in this subset of beneficiaries with new opioid use is difficult to judge from these data; however, recent evidence has not supported the effectiveness of long-term opioid therapy compared with nonopioid therapy for management of chronic pain and functional impairment among adults. ${ }^{25}$

Despite knowing that the risk of adverse outcomes increases with opioid dose, the exact increases in risk of opioid-related harms for older adults using CDC-defined thresholds is not known. ${ }^{26-28}$ Most research on the risk of opioid dose and 
opioid-related harms has been conducted in younger adult populations, even though painful conditions and opioid prescriptions are common among adults aged 65 years and older. ${ }^{1}$ Compared with younger adults, older adults are at additional risk for opioid-related harms because of a high incidence of comorbidities exacerbated by opioids, use of multiple medications with potential opioid interactions, fragmented care across multiple providers, and increased susceptibility to opioid-adverse drug effects. ${ }^{29-32}$

Unlike previous research that grouped acute and long-term opioid use, we identified individuals with new opioid use in order to examine the patterns that occur within the first year and are amenable to intervention by prescribing clinicians. ${ }^{11}$ The prescription patterns observed (i.e., long-term use, high doses, and multiple prescribers and dispensing pharmacies) can be particularly helpful to identify uncoordinated care and the potential for prescribing errors or opioid-related adverse events. Because MME measures directly represent the amount and timing of opioid exposure, we hypothesized that MME measures, whether peak or average MME values, may be a better reflection of risk than number of prescriptions. A recently implemented Medicare policy includes limits on the dose and duration of initial prescriptions for Part D beneficiaries, including a care coordination edit at the threshold of $90 \mathrm{MME}$ and options for plans to set pharmacy alerts at $200 \mathrm{MME}$ or to assess the presence of multiple prescribers and pharmacies. ${ }^{33}$

Our results suggest that interventions targeting beneficiaries with high opioid doses may also identify those with other risky use patterns. For clinicians, identifying the presence of multiple prescribers or pharmacies among those prescribed high doses may then indicate the context and appropriateness of the dose. Future work may look at networks of opioid prescribers to find opportunities to improve care coordination and communication between providers involved in the care of patients with complex needs.

Similarly, for managed care entities, which are a major force in front-line efforts to curb the crisis of opioid misuse, addiction, and overdose at the population level, it may be useful to understand trends in publicly funded health care, especially as Medicare managed care enrollment is increasing. ${ }^{34}$ Identifying and intervening in patient-level risk factors have been presented as a central strategy moving forward in efforts to prevent opioid misuse and addiction. ${ }^{35}$

This work provides initial evidence that a time-independent measure of peak MME may provide valuable insight for managed care companies developing risk prediction models to target intervention to improve opioid outcomes. This descriptive epidemiological work examined the lower MME thresholds aligned with CDC prescribing guidelines. In addition, our understanding of risk factors, such as having multiple opioid prescribers, will need refinement in the older adult population to correctly identify risk situations (e.g., concurrent unrelated opioid prescribers) and potential protective factors (e.g., care coordination). The CDC guidelines altered opioid prescribing rates and intensity; however, any specific effects among older adults have not been assessed and may benefit from the use of varying dose thresholds. ${ }^{36}$ These critical findings will enable us to improve existing policy to reduce risk from prescription opioids and better target those at risk for appropriate intervention.

\section{Limitations}

The results of this study should be considered in the context of the following limitations. Medicare prescription data are representative of individuals enrolled in stand-alone Part D prescription drug plans in 2014-2015 and may differ in terms of health status from managed care populations. ${ }^{37}$ Although we were unable to assess effects of the 2016 CDC guidelines, our results can serve as a benchmark for future evaluations. The prescription records reflect opioid medications that were paid for by Medicare and not those obtained fully out of pocket or by diversion or illicit means. The peak MME measure does not reflect the time-varying nature of intended opioid exposure nor do prescription claims allow us to know how prescribed opioids are actually being used.

In order to describe the general Medicare population with heterogeneous reasons for opioid use, we did not examine the indication for opioid use and did not exclude those with cancer or those with a single opioid prescription. We excluded individuals without complete follow-up data, including those who died, which could mask those who died as a result of opioid use. Finally, without data on pain severity and physical status, it is difficult to determine whether certain opioid prescription patterns are appropriate.

\section{Conclusions}

This study of Medicare beneficiaries aged 65 years and older reports that doses of opioids more than 50 MME (the lowest CDC guideline threshold) were prescribed in over one quarter of those with new opioid use in 2014. Having multiple opioid prescribers or multiple opioid-dispensing pharmacies was common, especially among those receiving doses of more than 50 MME per day. In addition, those receiving very high doses ( $\geq 90$ MME per day) were more likely to also have long-term use. To enable evidence-based policy and clinical practice changes from our results, future research will need to quantify the risks associated with these use patterns among older adults. 


\section{Authors}

SUDHA R. RAMAN, PhD, and ASHELEY C. SKINNER, PhD, Department of Population Health Sciences, Duke University School of Medicine, and Duke Clinical Research Institute, Duke University, Durham, North Carolina. CHRISTOPHER BUSH, MPH, Department of Population Health Sciences, Duke University School of Medicine, Durham, North Carolina. RUCHIR N. KARMALI, PhD, Department of Population Health Sciences, Duke University School of Medicine and Duke Clinical Research Institute, Duke University, Durham, North Carolina, and Division of Research, Kaiser Permanente Northern California, Oakland. LAWRENCE H. GREENBLATT, MD, Department of Medicine and Department of Community and Family Medicine, Duke University School of Medicine, Durham, North Carolina. ANDREW W. ROBERTS, PharmD, PhD, Department of Population Health and Department of Anesthesiology, University of Kansas Medical Center, Kansas City.

AUTHOR CORRESPONDENCE: Sudha R. Raman, PhD, Department of Population Health Sciences, Duke University, 215 Morris St., Duke Box 104023, Durham, NC 27701. Tel: 919.668.2690; E-mail: sudha.raman@duke.edu.

\section{DISCLOSURES}

No funding supported this study. Raman reports research grants from GlaxoSmithKline not related to this study. Roberts was supported by a CTSA grant from NCATS awarded to the University of Kansas Medical Center for Frontiers: The Heartland Institute for Clinical and Translational Research (\#KL2TR000119). The other authors have no potential conflicts to report.

\section{REFERENCES}

1. Patel KV, Guralnik JM, Dansie EJ, Turk DC. Prevalence and impact of pain among older adults in the United States: findings from the 2011 National Health and Aging Trends Study. Pain. 2013;154(12):2649-57.

2. Steinman MA, Komaiko KDR, Fung KZ, Ritchie CS. Use of opioids and other analgesics by older adults in the United States, 1999-2010. Pain Med. 2015;16(2):319-27.

3. Centers for Disease Control and Prevention. 2018 annual surveillance report of drug-related risks and outcomes-United States. Surveillance Special Report. August 31, 2018. Available at: https://www.cdc.gov/drugoverdose/pdf/pubs/2018-cdc-drug-surveillance-report.pdf. Accessed June 12, 2019.

4. Hedegaard H, Miniño AM, Warner M. Drug overdose deaths in the United States, 1999-2017. NCHS Data Brief No. 329. November 2018. Available at: https://www.cdc.gov/nchs/data/databriefs/db329-h.pdf. Accessed June 12, 2019.

5. Tadros A, Layman SM, Davis SM, Davidov DM, Cimino S. Emergency visits for prescription opioid poisonings. J Emerg Med. 2015;49(6):871-77.

6. Owens P, Barrett M, Weiss A, Washington R, Kronick R. Hospital inpatient utilization related to opioid overuse among adults, 1993-2012. Healthcare Cost and Utilization Project (HCUP). Statistical Brief \#177. July 2014. Available at: https://www.hcup-us.ahrq.gov/reports/statbriefs/sb177Hospitalizations-for-Opioid-Overuse.jsp. Accessed June 12, 2019.

7. West NA, Severtson SG, Green JL, Dart RC. Trends in abuse and misuse of prescription opioids among older adults. Drug Alcohol Depend. 2015;149:117-21.

8. Solomon DH, Rassen JA, Glynn RJ, et al. The comparative safety of opioids for nonmalignant pain in older adults. Arch Intern Med. 2010;170(22):1979-86
9. Jena AB, Goldman D, Weaver L, Karaca-Mandic P. Opioid prescribing by multiple providers in Medicare: retrospective observational study of insurance claims. BMJ. 2014;348:g1393.

10. Kuo YF, Raji MA, Chen NW, Hasan H, Goodwin JS. Trends in opioid prescriptions among Part D Medicare recipients from 2007 to 2012. Am J Med. 2016;129(2):221.e21-221.e30. Available at: https://www.amjmed.com/ article/S0002-9343(15)00999-7/fulltext. Accessed June 12, 2019.

11. Musich S, Wang SS, Slindee L, Kraemer S, Yeh CS. Prevalence and characteristics associated with high dose opioid users among older adults. Geriatr Nurs. 2019;40(1):31-36.

12. Axeen S. Trends in opioid use and prescribing in Medicare, 2006-2012 Health Serv Res. 2018;53(5):3309-28.

13. Adewumi AD, Hollingworth SA, Maravilla JC, Connor JP, Alati R. Prescribed dose of opioids and overdose: a systematic review and metaanalysis of unintentional prescription opioid overdose. CNS Drugs. 2018;32(2):101-16.

14. Hoadley J. Cubanski J, Neuman T. Medicare Part D in 2016 and trends over time. Henry J. Kaiser Family Foundation. September 16, 2016. Available at: https://www.kff.org/medicare/report/medicare-part-d-in-2016and-trends-over-time/. Accessed June 12, 2019.

15. Dunn KM, Saunders KW, Rutter CM, et al. Opioid prescriptions for chronic pain and overdose: a cohort study. Ann Intern Med. 2010;152(2):85-92.

16. Bennett M, Paice JA, Wallace M. Pain and opioids in cancer care: benefits, risks, and alternatives. Am Soc Clin Oncol Educ Book. 2017;37:705-13.

17. Loren AW. Harder to treat than leukemia-opioid use disorder in survivors of cancer. N Engl J Med. 2018;379(26):2485-87.

18. Carmichael AN, Morgan L, Del Fabbro E. Identifying and assessing the risk of opioid abuse in patients with cancer: an integrative review. Subst Abuse Rehabil. 2016;7:71-79.

19. Anghelescu DL, Ehrentraut JH, Faughnan LG. Opioid misuse and abuse: risk assessment and management in patients with cancer pain. J Natl Compr Canc Netw. 2013;11(8):1023-31.

20. Centers for Medicare \& Medicaid Services. Dual eligible beneficiaries under Medicare and Medicaid. MLN booklet. May 2018. Available at: https:// www.cms.gov/Outreach-and-Education/Medicare-Learning-Network-MLN/ MLNProducts/downloads/Medicare_Beneficiaries_Dual_Eligibles_At_a_ Glance.pdf. Accessed June 12, 2019.

21. Centers for Disease Control and Prevention. Analyzing prescription data and morphine milligram equivalents (MME). Updated April 10, 2019. Available at: https://www.cdc.gov/drugoverdose/resources/data.html. Accessed June 12, 2019.

22. Dowell D, Haegerich TM, Chou R. CDC guideline for prescribing opioids for chronic pain-United States, 2016. JAMA. 2016;315(15):1624-45. 23. Chou R, Fanciullo GJ, Fine PG, et al. Clinical guidelines for the use of chronic opioid therapy in chronic noncancer pain. J Pain. 2009;10(2):113-30.

24. American Geriatrics Society Panel on Pharmacological Management of Persistent Pain in Older Persons. Pharmacological management of persistent pain in older persons. J Am Geriatr Soc. 2009;57(8):1331-46.

25. Krebs EE, Gravely A, Nugent S, et al. Effect of opioid vs nonopioid medications on pain-related function in patients with chronic back pain or hip or knee osteoarthritis pain: the SPACE randomized clinical trial. JAMA. 2018;319(9):872-82.

26. Bohnert AS, Valenstein M, Bair MJ, et al. Association between opioid prescribing patterns and opioid overdose-related deaths. JAMA. 2011;305(13):1315-21.

27. Liang Y, Turner BJ. Assessing risk for drug overdose in a national cohort: role for both daily and total opioid dose? J Pain. 2015;16(4):318-25.

28. Saunders KW, Dunn KM, Merrill JO, et al. Relationship of opioid use and dosage levels to fractures in older chronic pain patients. J Gen Intern Med. 2010;25(4):310-15. 
29. Barile JP, Thompson WW, Zack MM, Krahn GL, Horner-Johnson W, Bowen SE. Multiple chronic medical conditions and health-related quality of life in older adults, 2004-2006. Prev Chronic Dis. 2013;10:E162

30. Steinman MA, Landefeld CS, Rosenthal GE, Berthenthal D, Sen S, Kaboli PJ. Polypharmacy and prescribing quality in older people. J Am Geriatr Soc. 2006;54(10):1516-23.

31. Holmes HM, Luo R, Kuo YF, Baillargeon J, Goodwin JS. Association of potentially inappropriate medication use with patient and prescriber characteristics in Medicare Part D. Pharmacoepidemiol Drug Saf. 2013;22(7):728-34.

32. El Desoky ES. Pharmacokinetic-pharmacodynamic crisis in the elderly. Am J Ther. 2007;14(5):488-98.

33. Centers for Medicare \& Medicaid Services. A prescriber's guide to the new Medicare Part D opioid overutilization policies for 2019. MLN Matters No. SE18016. November 1, 2018. Available at: https://www. cms.gov/Outreach-and-Education/Medicare-Learning-Network-MLN/ MLNMattersArticles/Downloads/se18016.pdf. Accessed June 12, 2019.
34. Centers for Medicare \& Medicaid Services. 2019 Medicare Advantage and Part D prescription drug program landscape. News release. September 28, 2018. Available at: https://www.cms.gov/newsroom/fact-sheets/2019medicare-advantage-and-part-d-prescription-drug-program-landscape. Accessed June 12, 2019.

35. Volkow ND, Jones EB, Einstein EB, Wargo EM. Prevention and treatment of opioid misuse and addiction: a review. JAMA Psychiatry. 2019;76(2):208-16.

36. Bohnert ASB, Guy GP Jr, Losby JL. Opioid prescribing in the United States before and after the Centers for Disease Control and Prevention's 2016 Opioid Guideline. Ann Intern Med. 2018;169(6):367-75.

37. Ndumele CD, Elliott MN, Haviland AM, et al. Differences in hospitalizations between fee-for-service and Medicare Advantage beneficiaries. Med Care. 2019;57(1):8-12 